\title{
Effect of Alfentanil on the Intraoperative Localization of an Epileptogenic Focus in Pediatric Patients with Intractable Seizure Disorder
}

\author{
${ }^{1}$ Department of Anesthesiology and Pain Medicine, ${ }^{2}$ Anesthesia and Pain Research Institute, \\ Yonsei University College of Medicine, Seoul, Korea
}

\author{
Byung In Choi, M.D. ${ }^{1}$, Sung Jin Lee, M.D. ${ }^{1,2}$, Bahn Lee, M.D. ${ }^{1}$, \\ Kyeong Tae Min, M.D. ${ }^{1,2}$, and Seung Ho Choi, M.D. ${ }^{1,2}$
}

\begin{abstract}
Background: Intraoperative alfentanil is known to increase the epileptic discharge in the adult seizure patients. This study hypothesized that alfentanil might localize an epileptogenic focus in pediatric seizure patients.

Methods: This study was performed in the pediatric seizure patients who undergo second-staged operation. Thirteen pediatric patients were enrolled and their epileptic foci were already suspected from first operation. After anesthetic induction, sevoflurane was maintained at $0.6 \%$ end-tidal concentration for study period. Electrocorticography (ECoG) was recorded for 5 minutes before and 5 minutes after alfentanil $20 \mu \mathrm{g} / \mathrm{kg}$ IV. During the ECoG recordings, the mean arterial pressure (MAP) and heart rate (HR) were continuously monitored. After the surgery, a pediatric neurologist analyzed the changes of spike frequencies of suspected ictal zone. The spike frequencies of suspected non-ictal zone were also calculated in five patients. The suspected ictal zone was defined as the most abundant spontaneous spiking area observed after first staged grid insertion.

Results: Alfentanil induced a significant increase in spike activity of suspected ictal zone in 12 out of 13 patients (median of 20 [ranged 10 to 100] vs 38 [ranged 20 to 100], $\mathrm{P}<0.05$ ). Alfentanil-induced increase of spike activity was prominent in the suspected ictal zone rather than non-ictal zone. There were no significant changes in the MAP and HR after administration of alfentanil.

Conclusions: Alfentanil activates epileptiform activity of suspected ictal zone in pediatric patients with seizure disorder and can be used to assist in the localization of the epileptogenic focus during seizure surgery. (Korean $\mathbf{J}$ Anesthesiol 2007; 52: $\mathbf{S}$ 47 52)
\end{abstract}

Key Words: alfentanil, electrocorticography, pediatric, seizure.

\section{INTRODUCTION}

Patients with intractable seizures may be amendable to surgical treatment to eliminate or decrease the frequency of their seizures. Surgical treatment involves the resection of a seizure focus or interruption of seizure circuits. It is commonly performed as a two-staged procedure. Grid insertion surgery is performed to determine the location of the seizure focus during daily living life. Thereafter, excision of a suspe-

Received : September 22, 2006

Corresponding to : Seung Ho Choi, Department of Anesthesiology and Pain Medicine, Severance Hospital, Yonsei University College of Medicine, 134, Shinchon-dong, Seodaemun-gu, Seoul 120-752, Korea. Tel: 822-2228-2431, Fax: 82-2-312-7185, E-mail: csho99@yumc.yonsei.ac.kr cted epileptogenic focus or interruption of seizure circuit is usually performed within two weeks. Intraoperative localization of a seizure focus is performed with electrocorticography (ECoG) using electrodes placed directly on the exposed brain. ${ }^{1)}$ The purposes of intraoperative ECoG are to identify the location of the epileptogenic zone, to avoid the resection of nonepileptogenic brain tissue, and to determine the adequacy of the surgical resection. ${ }^{2}$

Most anesthetics suppress the cortical activities, and it is not easy to identify the suspected ictal area under conventional surgical level of anesthesia. Therefore, it is often necessary to chemically stimulate the epileptogenic activity to aid in the localization of the epileptogenic focus. Many drugs have been used to activate epileptiform activity, including methohexital, propofol, sevoflurane and ketamine. ${ }^{3-6)}$ Opioids are often used 
to localize the epileptogenic focus in adult patients. ${ }^{7-10)}$ For example, alfentanil facilitates intraoperative ECoG-guided localization of the epileptogenic focus during seizure surgery. ${ }^{11-13)}$ However, the effect of alfentanil on ECoG in pediatric patients with seizure disorder has not been studied. The etiology, pharmacologic responses and prognosis of pediatric seizure patients are different from those of adult patients.

The aim of this study was to investigate the effect of alfentanil on the intraoperative localization of an epileptogenic focus in pediatric patients with intractable seizure disorder.

\section{MATERIALS AND METHODS}

After approval from the institutional review board and informed consents from the patients, thirteen pediatric patients undergoing second-staged surgical resection of an epileptogenic focus were enrolled in this study. They underwent first-staged subdural grid insertion surgery within two weeks before second-staged operation. The patients were allowed to take their anticonvulsant medications until the time of surgery.

No premedication was administered. All patients were monitored with electrocardiography, arterial blood pressure and pulse oximetry during the operation. The anesthetic technique was standardized. Anesthesia was induced with thiopental 5 $\mathrm{mg} / \mathrm{kg}$, and rocuronium $0.6 \mathrm{mg} / \mathrm{kg}$ was given intravenously for neuromuscular blockade. After tracheal intubation, the lungs were ventilated to maintain $\mathrm{Et}_{\mathrm{CO} 2}$ between $30-35 \mathrm{mmHg}$.
Anesthesia was maintained with sevoflurane, 50\% oxygen in air and additional doses of rocuronium as necessary. The patient's temperature was maintained within normal limits.

After induction, sevoflurane was maintained at $0.6 \%$ endtidal concentration at least for $10 \mathrm{~min}$ before the study. During the study period, any surgical manipulations were not allowed. After $5 \mathrm{~min}$ of steady-state recording for a baseline ECoG, each patient received alfentanil $20 \mathrm{\mu g} / \mathrm{kg} \mathrm{IV}$, followed by $5 \mathrm{~min}$ of recording of ECoG after the administration of alfentanil. During the ECoG recordings, the mean arterial pressure (MAP) and heart rate (HR) were continuously monitored and documented for $5 \mathrm{~min}$ before and after alfentanil administration. Multi-channel analog ECoG recordings from the previously implanted subdural electrodes using an EEG$4421 \mathrm{k}^{\mathrm{TM}}$ (Nihon Kohden Corp., Tokyo, Japan) were recorded on paper by a pen-recorder. We were informed of the suspected ictal area from a pediatric neurologist. The pediatric neurologist was not allowed to participate in study period. The ECoG recordings before and after alfentanil administration were coded by unintended characters (Fig. 1). The most abundant spontaneous spiking area during awaken state at ward after first staged grid insertion was defined as the suspected ictal zone.

After the surgery, a pediatric neurologist analyzed the ECoG recordings and calculated the spike frequencies of suspected ictal zone before and after the administration of alfentanil. The spike frequencies of suspected non-ictal zone were also calculated in five patients.
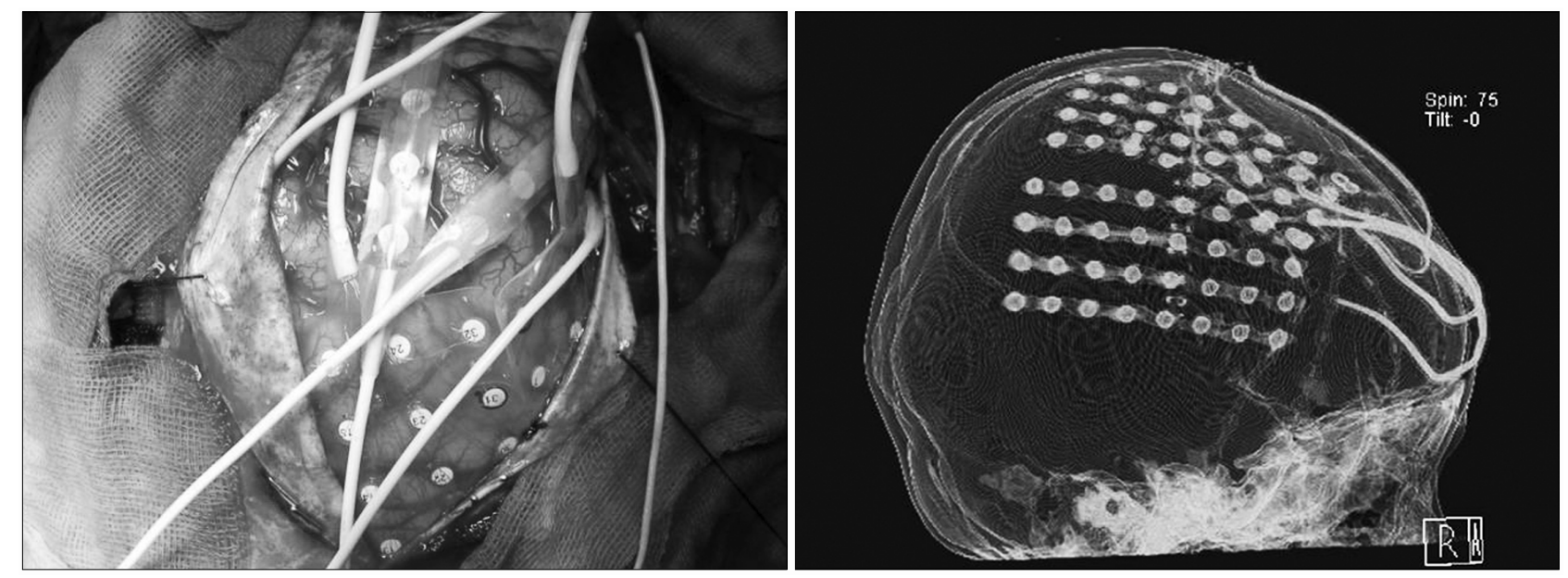

Fig. 1. Intraoperative electrocorticographic (ECoG) electrodes. ECoG electrodes have been placed over the brain surface covering suspected ictal zone at the time of first-staged grid insertion surgery. 
Table 1. Demographic Data

\begin{tabular}{crrll}
\hline $\begin{array}{c}\text { Patient } \\
\text { number }\end{array}$ & Sex & $\begin{array}{c}\text { Age } \\
\text { (yr) }\end{array}$ & \multicolumn{1}{c}{ Diagnosis } & \multicolumn{1}{c}{ Operation name } \\
\hline 1 & M & 16 & Hippocampal sclerosis & Temporalobectomy \& cortisectomy \\
2 & $\mathrm{M}$ & 8 & Lennox-Gastaut syndrome & Temporal lobectomy \& hippocampectomy \\
3 & $\mathrm{M}$ & 14 & Lennox-Gastaut syndrome & Frontal lobectomy \\
4 & $\mathrm{M}$ & 7 & Cortical dysplasia & Frontal lobectomy \& cortisectomy \\
5 & $\mathrm{M}$ & 2 & Complex partial seizure & Frontoparietal hemispherectomy \\
6 & $\mathrm{~F}$ & 9 & Myoclonic seizure & Frontal lobectomy \& callosotomy \\
7 & $\mathrm{M}$ & 7 & Lennox-Gastaut syndrome & Callosotomy \\
8 & $\mathrm{M}$ & 3 & Complex partial seizure & Frontal lobectomy \\
9 & $\mathrm{~F}$ & 10 & Complex partial seizure & Frontal lobectomy \\
10 & $\mathrm{~F}$ & 11 & Complex partial seizure & Temporal lobectomy \\
11 & $\mathrm{~F}$ & 15 & Complex partial seizure & Temporal lobectomy \\
12 & $\mathrm{M}$ & 9 & Myoclonic seizure & Frontal lobectomy \& callosotomy \\
13 & $\mathrm{M}$ & 4 & Infantile spasm & Frontal lobectomy \\
\hline
\end{tabular}

Table 2. The Effects of Alfentanil on the Spike Activity, Mean Arterial Pressure and Heart Rate

\begin{tabular}{|c|c|c|}
\hline & \multicolumn{2}{|c|}{ Alfentanil $(20 \mu \mathrm{g} / \mathrm{kg})$} \\
\hline & Before & After \\
\hline Spike activity (number/min) & $20(10-100)$ & $38 *(20-100)$ \\
\hline Mean arterial pressure $(\mathrm{mmHg})$ & $78 \pm 22$ & $76 \pm 20$ \\
\hline Heart rate (beats/min) & $103 \pm 14$ & $99 \pm 17$ \\
\hline
\end{tabular}

Values are median number (range) or mean \pm SD.

* $\mathrm{P}<0.05$ compared to the values measured before the administration of alfentanil.

Changes in MAP and HR were analyzed using the paired t-test. Comparisons of the changes of spike frequencies of suspected ictal zone before and after the administration of alfentanil were performed using Wilcoxon signed ranks test. $\mathrm{P}<0.05$ was considered significant.

\section{RESULTS}

Demographic data of 13 patients were shown in Table 1 . Alfentanil induced a significant increase in spike activity in 12 out of 13 patients in suspected ictal zone (median value of 20 [ranged 10 to 100] vs 38 [ranged 20 to 100], $\mathrm{P}<0.05$ ) (Table 2) (Fig. 2). All patients showed spontaneous spikes before the administration of alfentanil in suspected ictal zone. The site of maximal activation induced by alfentanil was

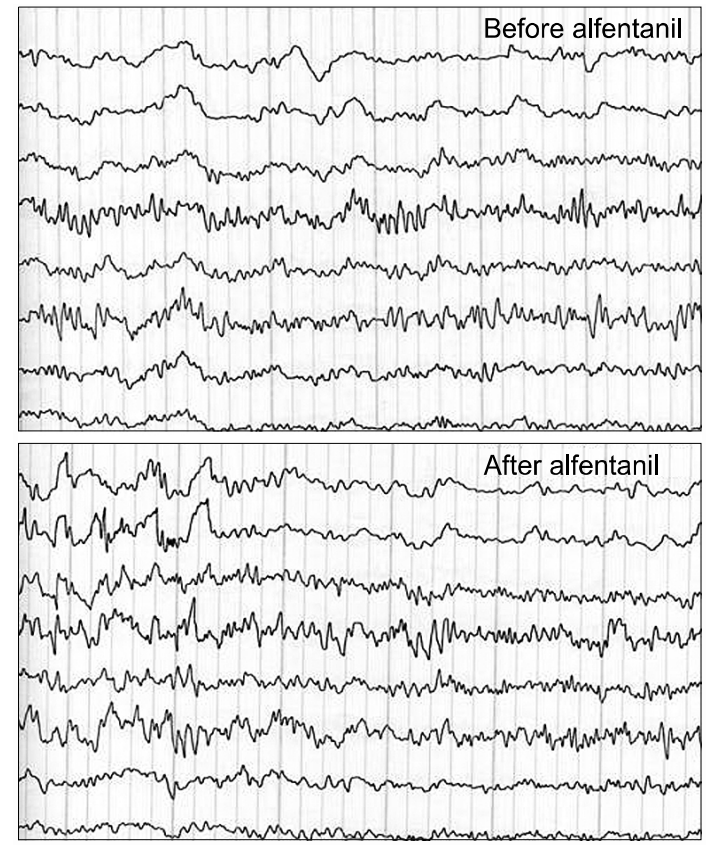

Fig. 2. Representative electrocorticography (ECoG) recordings obtained before and after alfentanil administration in patient number 5 . Alfentanil induces a significant increase in spike activity in suspected ictal zone (upper 6 channels) but not in non-ictal zone (lower 2 channels). Upper 6 channels were recorded in frontal lobe, and lower 2 channels were recorded in the area $2 \mathrm{~cm}$ off from suspected ictal zone.

identical to the suspected ictal zone. Alfentanil-induced increase of spike activity was prominent in the suspected ictal zone 
rather than non-ictal zone (Fig. 2). No patient had electrographic or clinical seizure activity. There were no significant changes in MAP and HR after the administration of alfentanil (Table 2).

\section{DISCUSSION}

In this study, we investigated the effect of alfentanil on the intraoperative localization of an epileptogenic focus in pediatric patients with intractable seizure disorder. Intraoperative administration of alfentanil induced a significant increase in spike activity in suspected ictal zone.

Patients with seizures refractory to medical therapy may undergo surgical resection of a seizure focus or interruption of seizure circuits in an attempt to control their epilepsy. Accurate identification of the epileptogenic focus or surgical resection margin is important. Therefore, resection of an epileptogenic focus is often performed by ECoG monitoring. The obvious advantages of the intraoperative ECoG are to offer flexible placement of recording electrodes, to be performed before and after surgery to assess the presence or absence of epileptiform activity, and to allow direct electrical stimulation of the brain so that the regions involved in functions that must be spared by the resection can be delineated with a high degree of confidence. ${ }^{2)}$ However, getting the precise information from ECoG is not easy during general anesthesia, as the anesthetics influence the recordings by suppressing electrical brain activity.

Opioids sometimes cause signs of neuroexcitation such as nystagmus, muscle rigidity, myoclonus, and seizure-like activity. ${ }^{14)}$ Hence, opioids have been candidates for evoking epileptic activity to identify epileptogenic foci.

Alfentanil is a rapidly acting opioid analgesic with short duration of action and is often used to facilitate intraoperative ECoG-guided localization of the epileptogenic zone for adult patients. $^{12,13)}$ Cascino et al. ${ }^{12)}$ retrospectively studied the effects of IV alfentanil $50 \mu \mathrm{g} / \mathrm{kg}$ in 23 adult patients during surgery for intractable epilepsy under general anesthesia. They found an increased frequency of interictal epileptiform discharges in 20 patients, and the maximal effect of alfentanil occurred at $30 \mathrm{sec}$ to $3 \mathrm{~min}$ after the administration of the drug. Our study showed that alfentanil $20 \mu \mathrm{g} / \mathrm{kg}$ produced a similar degree of ECoG activation in pediatric patients.

Another more recent article reported the effect of alfentanil activation of epileptiform activity in children. Keene et al. ${ }^{13)}$ noted that alfentanil $20 \mu \mathrm{g} / \mathrm{kg}$ induced epileptiform activity in $83 \%$ of patients, and $25 \%$ of these patients had electrographic seizures. Many of these patients had mesial temporal foci, and epileptiform abnormalities appeared within $5 \mathrm{~min}$ in all cases. Our study showed that many of patients had frontal foci and no evidence of seizure activity was detected. Although we did not estimate the spike frequencies of suspected ictal and non-ictal zone in all patients, alfentanil- induced increase of spike activity was prominent in the suspected ictal zone rather than non-ictal zone.

Alfentanil is often used in anesthesia in a wide range of doses. Although the activation effect of alfentanil in epileptic patients is well documented, alfentanil does not seem to exert similar effect on the EEG of normal individuals. Bovill et al. ${ }^{15)}$ did not observe electroencephalographic activation of epileptiform potentials following the intraoperative administration of alfentanil even with the dose of $120 \mu \mathrm{g} / \mathrm{kg}$ IV into patients undergoing carotid endarterectomy.

Opioids can induce electrographic seizures during ECoG recordings in patients with epilepsy. Although the results of the previous studies confirm that remifentanil, alfentanil and fentanyl all activate epileptic discharges, ${ }^{10,11)}$ these opioids in the doses employed had different effects on the epileptic threshold, and alfentanil seems to have more pronounced epileptogenic effects. ${ }^{16)}$ Manninen et al. $^{11)}$ showed that two patients had electrographic evidence of seizure activity after the administration of alfentanil $50 \mu \mathrm{g} / \mathrm{kg}$. Contrary to them, we did not detect any evidence of ECoG or clinical seizure activity during the study periods with a dose of alfentanil 20 $\mu \mathrm{g} / \mathrm{kg}$ in pediatric patients.

Sevoflurane is often used during neurosurgical procedures. For successful intraoperative ECoG monitoring, it is important for anesthesiologists to prevent the patient from intraoperative awareness as well as to minimize the effects of anesthetics on intraoperative monitorings. Kihara et al. ${ }^{17)}$ showed that the minimum alveolar concentration (MAC)-awake of sevoflurane was observed to be 0.78 vol. $\%$ in children, and oral clonidine premedication of $2 \mu \mathrm{g} / \mathrm{kg}$ reduced the MAC-awake to 0.36 vol.\%. Watts et al. ${ }^{18)}$ suggested that the capacity to modulate neuroexcitability is a dose-dependent feature of volatile anesthetic that is manifested most prominently at near burstsuppression doses (i.e., 1.5 times the MAC) and is minimal or absent at low doses. In our study, sevoflurane was maintained at $0.6 \%$ end-tidal concentration during the study periods of 10 min and alfentanil $20 \mu \mathrm{g} / \mathrm{kg}$ IV was administered before the 
recording. Therefore, intraoperative awareness and the effect of sevoflurane on the ECoG might be little concerned in our study.

The mechanisms underlying opioid-induced epileptogenic activity are poorly understood. Experimental evidence suggests that induction of epileptiform activity may be mediated through activation of multiple opioid receptor subtypes. ${ }^{19)}$ A separate mechanism of activation may be mediated by opiate antagonism of inhibitory GABAergic neurotransmission. ${ }^{20-22)}$ Zhang et al. $^{23)}$ showed that methohexital potentiates GABAmediated synaptic inhibition and likely activates epileptiform activity at ECoG through a resulting increase in neuronal synchrony. The similar activation in interictal epileptiform activity seen at ECoG with opiates and barbiturates is interesting in that multiple mechanisms may underlie human epileptogenesis. Kofke et al. ${ }^{24)}$ suggested that high doses of alfentanil administered to rats can produce limbic system seizure activity with hypermetabolism associated with neuropathologic lesions.

Surgical treatment for intractable seizure disorder is commonly performed on two-staged surgery for the precise detection of epileptogenic foci. If intraoperative alfentanil confers the capability of the localization of interictal area, it would be meaningful tool during one-staged operation in pediatric patients. However, it is unclear that alfentanil would increase the spike activities of epileptogenic foci regardless of the etiologies or anatomic locations. Furthermore, alfentanil may still act as proconvulsant. $^{25)}$

In conclusion, we showed that alfentanil activates epileptiform activity in pediatric patients with intractable seizure disorder. Alfentanil can be used to assist in the localization of the epileptogenic focus during seizure surgery.

\section{REFERENCES}

1. Ojemann GA: Different approaches to resective epilepsy surgery: standard and tailored. Epilepsy Res Suppl 1992; 5: 169-74.

2. Kuruvilla A, Flink R: Intraoperative electrocorticography in epilepsy surgery: useful or not? Seizure 2003; 12: 577-84.

3. Kofke WA, Tempelhoff R, Dasheiff RM: Anesthetic implications of epilepsy, status epilepticus, and epilepsy surgery. J Neurosurg Anesthesiol 1997; 9: 349-72.

4. Park SI, Shin YS, Ji JN, Yoon HS, Kim YH, Son SC, et al: Myoclonic and tonic-clonic seizures after propofol sedation during a brachial plexus block: A case report. Korean J Anesthesiol 2003; 45: $544-6$.

5. Iijima $T$, Nakamura $Z$, Iwao $Y$, Sankawa $H$ : The epileptogenic properties of the volatile anesthetics sevoflurane and isoflurane in patients with epilepsy. Anesth Analg 2000; 91: 989-95.

6. Jaaskelainen SK, Kaisti K, Suni L, Hinkka S, Scheinin H: Sevoflurane is epileptogenic in healthy subjects at surgical levels of anesthesia. Neurology 2003; 61: 1073-8.

7. Tommasino C, Maekawa T, Shapiro HM, Keifer-Goodman J, Kohlenberger RW: Fentanyl-induced seizures activate subcortical brain metabolism. Anesthesiology 1984; 60: 283-90.

8. Tempelhoff R, Modica PA, Bernardo KL, Edwards I: Fentanyl-induced electrocorticographic seizures in patients with complex partial epilepsy. J Neurosurg 1992; 77: 201-8.

9. Ross J, Kearse LA Jr, Barlow MK, Houghton KJ, Cosgrove GR: Alfentanil-induced epileptiform activity: a simultaneous surface and depth electroencephalographic study in complex partial epilepsy. Epilepsia 2001; 42: 220-5.

10. Wass CT, Grady RE, Fessler AJ, Cascino GD, Lozada L, Bechtle PS, et al: The effects of remifentanil on epileptiform discharges during intraoperative electrocorticography in patients undergoing epilepsy surgery. Epilepsia 2001; 42: 1340-4.

11. Manninen PH, Burke SJ, Wennberg R, Lozano AM, El Beheiry $\mathrm{H}$ : Intraoperative localization of an epileptogenic focus with alfentanil and fentanyl. Anesth Analg 1999; 88: 1101-6.

12. Cascino GD, So EL, Sharbrough FW, Strelow D, Lagerlund TD, Milde LN, et al: Alfentanil-induced epileptiform activity in patients with partial epilepsy. J Clin Neurophysiol 1993; 10: 520-5.

13. Keene DL, Roberts D, Splinter WM, Higgins M, Ventureyra E: Alfentanil mediated activation of epileptiform activity in the electrocorticogram during resection of epileptogenic foci. Can J Neurol Sci 1997; 24: 37-9.

14. Scott JC, Sarnquist FH: Seizure-like movements during a fentanyl infusion with absence of seizure activity in a simultaneous EEG recording. Anesthesiology 1985; 62: 812-4.

15. Bovill JG, Sebel PS, Wauquier A, Rog P, Schuyt HC: Influence of high-dose alfentanil anaesthesia on the electroencephalogram: correlation with plasma concentrations. Br J Anaesth 1983; 55: S 199-209.

16. McGuire G, El-Beheiry H, Manninen P, Lozano A, Wennberg R: Activation of electrocorticographic activity with remifentanil and alfentanil during neurosurgical excision of epileptogenic focus. $\mathrm{Br}$ J Anaesth 2003; 91: 651-5.

17. Kihara S, Inomata S, Yaguchi $Y$, Toyooka H, Baba $Y$, Kohda $Y$ : The awakening concentration of sevoflurane in children. Anesth Analg 2000; 91: 305-8.

18. Watts AD, Herrick IA, McLachlan RS, Craen RA, Gelb AW: The effect of sevoflurane and isoflurane anesthesia on interictal spike activity among patients with refractory epilepsy. Anesth Analg 1999; 89: 1275-81.

19. Zhu H, Zhou W: Morphine induces synchronous oscillatory discharges in the rat locus coeruleus. J Neurosci 2001; 21: RC 179.

20. Zieglgansberger W, French ED, Siggins GR, Bloom FE: Opioid peptides may excite hippocampal pyramidal neurons by inhibiting adjacent inhibitory interneurons. Science 1979; 205: 415-7.

21. Werz MA, Macdonald RL: Opiate alkaloids antagonize postsy- 
Korean J Anesthesiol : Vol. 52. No. 6, 2007

naptic glycine and GABA responses: correlation with convulsant action. Brain Res 1982; 236: 107-19.

22. Frenk H: Pro-and anticonvulsant actions of morphine and the endogenous opioids: involvement and interactions of multiple opiate and non-opiate systems. Brain Res 1983; 6: 197-210.

23. Zhang L, Zhang Y, Wennberg R: Multiple actions of methohexital on hippocampal CA1 and cortical neurons of rat brain slices. J
Pharmacol Exp Ther 1998; 286: 1177-82.

24. Kofke WA, Garman RH, Tom WC, Rose ME, Hawkins RA: Alfentanil-induced hypermetabolism, seizure, and histopathology in rat brain. Anesth Analg 1992; 75: 953-64.

25. Strong WE, Matson M: Probable seizure after alfentanil. Anesth Analg 1989; 68: 692-3. 